\title{
Respuesta hormonal adaptativa en atletas durante la práctica del voleibol de piso. Una aproximación desde la endocrinología del deporte
}

\section{Adaptative hormonal response in athletes during indoor volleyball practice. An approach from sports endocrinology}

\author{
Jorge Andrés Barrero (iD; María Alejandra Barrero-Casallas² iD
}

'Universidad Nacional de Colombia, Facultad de Medicina. Bogotá D.C, Colombia. jobarreroc@unal.edu.co ${ }^{2}$ Universidad de La Sabana, Facultad de Medicina. Chía - Cundinamarca, Colombia. mariabarca@unisabana.edu.co

Cómo citar: Barrero, J.A.; Barrero-Casallas, M.A. 2022. Respuesta hormonal adaptativa en atletas durante la práctica del voleibol de piso. Una aproximación desde la endocrinología del deporte. Rev. Digit. Act. Fis. Deport. 8(1):e2031. http://doi.org/10.31910/rdafd. v8.n1.2022.2031

Artículo de acceso abierto publicado por Revista Digital: Actividad Física y Deporte, bajo una licencia Creative Commons CC BY-NC 4.0

Publicación oficial de la Universidad de Ciencias Aplicadas y Ambientales U.D.C.A, Institución de Educación Superior Acreditada de Alta Calidad por el Ministerio de Educación Nacional.

Recibido: julio 7 de 2021 Aceptado: noviembre 4 de 2021 Editado por: Álvaro José Gracia Díaz

\section{RESUMEN}

Introducción: Los cambios en el patrón de secreción hormonal constituyen uno de los principales mecanismos de regulación homeostática en respuesta al ejercicio físico. Pese a que en los deportes de contacto se ha observado un aumento considerable en los marcadores séricos de estrés, la ausencia de contacto físico entre jugadores genera incertidumbre frente a la posibilidad de que la respuesta endocrina, durante la práctica del voleibol de piso, sea poco significativa. Objetivo: La presente investigación desarrolla una revisión bibliográfica de la respuesta hormonal adaptativa en voleibolistas antes, durante y posterior a sesiones de práctica de voleibol de piso, así como en condiciones basales, en comparación con sujetos sedentarios. Metodología: Se llevó a cabo una revisión bibliográfica en las bases de datos PubMed, Scopus y ScienceDirect de estudios reportados hasta junio del 2021. Resultados: Diez estudios fueron incluidos, a partir de los cuales, se observó que los voleibolistas exhiben niveles elevados de cortisol, testosterona y $\mathrm{GH}$, posterior a sesiones de práctica. Los cambios en la concentración de IGF-1 al igual que el efecto del entrenamiento sobre la respuesta endocrina, mostraron resultados contradictorios. Conclusiones: Aún cuando el cortisol, la testosterona y la GH aumentan después de sesiones de juego, este incremento parece ser mayor en las primeras semanas de temporada de competencia para el cortisol y mayor en las últimas semanas, para la testosterona y la GH. Determinar si el entrenamiento regular logra modular la respuesta endocrina durante las sesiones de juego de voleibol de piso, permanece sujeto a futuras investigaciones.

Palabras clave: Voleibol; Cortisol; Testosterona; Hormona del Crecimiento; Factor 1 del Crecimiento Similar a la Insulina.

\section{ABSTRACT}

Introduction: Changes in the hormonal secretion pattern constitute one of the main homeostatic regulatory mechanisms in response to physical activity. Although a considerable increase in serum stress markers has been documented during contact sports practice, the absence of physical contact between volleyball players generates uncertainty about the possibility that the endocrine response during indoor 
volleyball practice may not be significant. Objective: This investigation develops a review of the adaptive hormonal response in volleyball players before, during and/or after indoor volleyball practice sessions as well as in basal conditions in comparison with sedentary subjects. Methodology: A literature review was conducted in PubMed, Scopus and ScienceDirect databases covering studies reported until June 2021. Results: Ten studies were included, from which it was observed that volleyball players exhibit elevated levels of cortisol, testosterone and $\mathrm{GH}$ following practice sessions. Changes in IGF-1 concentration as well as the effect of training on endocrine response showed conflicting results. Conclusion: Although cortisol, testosterone and $\mathrm{GH}$ increase after practice sessions, this increase seems higher in the first weeks of the competition season for cortisol, and in the last weeks for testosterone and $\mathrm{GH}$. Whether regular training is successful in modulating the endocrine response during indoor volleyball practice sessions remains subject to future research.

Keywords: Volleyball; Cortisol; Testosterone; Growth Hormone; Insulin-like Growth Factor 1.

\section{INTRODUCCIÓN}

La práctica deportiva conlleva una alta demanda biomecánica y un estado de estrés físico, al cual responde el sistema endocrino, como parte de los mecanismos fisiológicos de regulación homeostática. Los cambios en el patrón de secreción hormonal durante el ejercicio reflejan la acción de los marcadores séricos de estrés, que confluyen en los diferentes circuitos neuroendocrinos, y que articulan una respuesta metabólica al aumento del gasto energético (Budde et al. 2016). En consecuencia, el ejercicio modifica la liberación de factores hipofisiotróficos y, subsecuentemente, la activación de ejes centrales como el hipotálamo-hipofisiario-adrenal, hipotálamohipofisiario-gonadal y el somatotropo (GH/IGF-1) (Kraemer et al. 2020). De igual forma, la actividad física modula sistemas periféricos, como el eje reninaangiotensina-aldosterona y la producción suprarrenal de catecolaminas (Zouhal et al. 2008; Nunes-Silva et al. 2017). Así, se entiende que los mecanismos de secreción hormonal muestran una importante adaptación a los periodos de esfuerzo físico y constituyen un pilar fundamental en la integración de la respuesta fisiológica al ejercicio (McGee \& Hargreaves, 2020).
El balance de hormonas anabólicas y catabólicas ha cobrado una gran relevancia como biomarcador de intensidad y demanda metabólica; particularmente, la proporción de testosterona/cortisol (T/C) se ha propuesto como un indicador del síndrome de sobreentrenamiento (Meeusen et al. 2013). Las variaciones en la razón $\mathrm{T} / \mathrm{C}$, se han asociado con una pérdida de electrolitos en la sudoración y la afectación del desempeño cognitivo (Castro-Sepúlveda et al. 2019; Caccese et al. 2021), lo que pone en evidencia el papel crucial de las hormonas en el rendimiento deportivo. Por otro lado, el entrenamiento excesivo crónico se ha vinculado con una inapropiada actividad secretora de diversas glándulas endocrinas, predisponiendo a estados de hipersecreción y producción hormonal inadecuada (Hackney \& Lane, 2015).

El consumo máximo de oxígeno (VOmax) refleja la intensidad del ejercicio físico y resulta de particular relevancia para la endocrinología del deporte, pues se ha documentado que establece el umbral de respuesta hormonal a la actividad física (Urhausen et al. 1995). Al evaluar el perfil catabólico, a partir de los niveles de glucocorticoides, Hill et al. (2008) demostraron que la actividad física de intensidad elevada (VOmax $>80 \%$ ) aumenta de forma significativa la concentración sérica de cortisol, mientras que el ejercicio de intensidad leve (VOmax<40\%), no muestra un incremento importante. Por otra parte, durante el ejercicio de resistencia, se ha descrito un aumento en los niveles de hormonas anabólicas como la testosterona, la hormona de crecimiento (GH) y el factor de crecimiento similar a la insulina tipo 1 (IGF-1) (Constantini \& Hackney, 2013). Es correcto suponer, entonces, que la intensidad del ejercicio y el entrenamiento de resistencia modulan, considerablemente, la respuesta endocrina en los atletas.

El voleibol de piso es un deporte que exige una compleja combinación de fuerza, potencia y agilidad. El jugador requiere una coordinación y un equilibrio dinámico, que le permita adecuarse a la demanda cognitiva y motora del control del balón (Hómez et al. 2017). A su vez, el voleibol implica cortas sesiones de esfuerzo de alta intensidad, a menudo, con poco tiempo de descanso entre partidos (Closs et al. 2020). No obstante, la ausencia de choques e impactos entre jugadores supone que el estrés físico que experimentan los voleibolistas puede resultar menor 
frente a aquellos atletas que practican deportes de contacto. En concordancia, Souglis et al. (2015) hallaron que los deportistas practicantes de voleibol exhiben el menor incremento en marcadores séricos de inflamación y daño muscular, al compararse con jugadores de fútbol y de baloncesto. En este sentido, persiste cierta incertidumbre frente a la posibilidad que la respuesta endocrina durante la práctica del voleibol de piso, al igual que la elevación de los marcadores de inflamación y daño muscular, sea poco significativa.

A conocimiento de los autores, a la fecha no se reporta un estudio que recopile la evidencia referente a la respuesta endocrina de tipo anabólica y catabólica en la práctica del voleibol. Por consiguiente, la presente investigación busca realizar una revisión bibliográfica de la respuesta hormonal adaptativa en voleibolistas, antes, durante y posterior a sesiones de práctica de voleibol de piso, así como en condiciones basales, en comparación con sujetos sedentarios.

\section{MATERIALES Y MÉTODOS}

Con base en los parámetros PRISMA (Moher et al. 2009), el presente estudio llevó a cabo una revisión de la literatura referente a los cambios en los niveles de hormonas catabólicas y anabólicas, en jugadores de voleibol en condiciones basales y en respuesta a sesiones de práctica o periodos de entrenamiento de

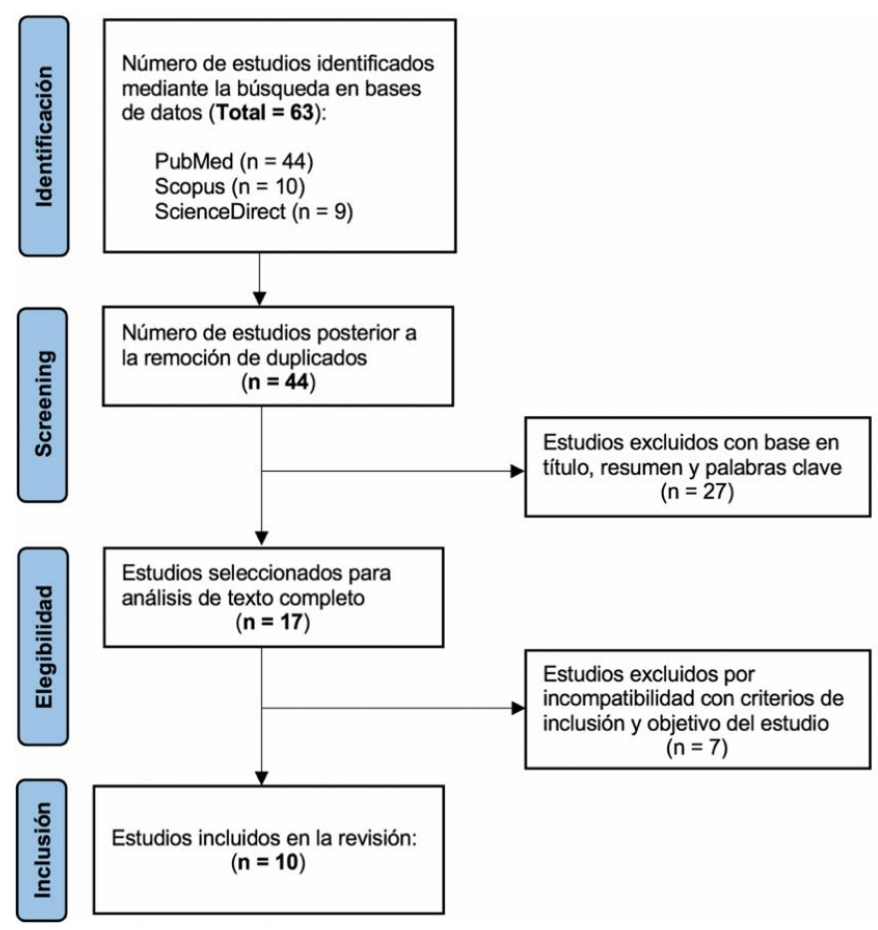

Figura 1. Flujograma de selección de estudios voleibol de piso. Para la elaboración de la estrategia de búsqueda, se indagaron por título, resumen y palabras clave, los siguientes términos: (1) Hormonas ("Testosterone" OR "Cortisol" OR "IGF-1" OR "GH" OR "Anabolic hormones" OR "Catabolic hormones") y (2) Voleibol ("Volleyball" OR "Volleyball players"). En la búsqueda bibliográfica, se consultaron las bases de datos PubMed, Scopus y ScienceDirect. La revisión abarca artículos de investigación publicados hasta junio del 2021.

Se incluyeron investigaciones con base en los siguientes criterios de inclusión: artículos de investigación original, escritos en idioma inglés y publicados en un rango de tiempo comprendido entre 2000-2021. La selección de cada artículo elegible fue debatida y aprobada por ambos autores. Posteriormente, de los estudios incluidos, se extrajeron los siguientes datos: (a) Autoría y año de publicación, (b) Población de estudio, (c) Diseño experimental y (d) Resultados relevantes.

Se recolectaron un total de 63 estudios, de los cuales, se evaluaron 49 posterior a la eliminación de duplicados. Consiguientemente, estos 49 artículos fueron filtrados con base en título y resumen y se obtuvo 17 estudios. Se procedió a realizar la lectura completa de los artículos, excluyendo aquellos inconsistentes con los criterios de inclusión. Finalmente, se incluyeron 10 estudios en la presente revisión. La figura 1 ilustra el proceso de selección de artículos. 


\section{RESULTADOS Y DISCUSIÓN}

La respuesta hormonal en voleibolistas se ha estudiado, a partir de la medición de los niveles séricos, salivales y urinarios, de diferentes hormonas y marcadores de estrés. En la presente revisión, se encontró un mayor número de estudios enfocados en la medición de cortisol y testosterona, así como de la razón T/C. Por otro lado, los niveles de hormonas como la GH e IGF-1 mostraron ser blanco de estudio, principalmente, en poblaciones en edades prepuberales y en jugadores de categoría juvenil. Los resultados de los estudios incluidos en la revisión se presentan en la tabla 1.

Tabla 1. Estudios incluidos en la revisión.

\begin{tabular}{|c|c|c|c|}
\hline Autor(es) (año) & Población de estudio & Diseño experimental & Resultados relevantes \\
\hline Roli et al. (2018) & $\begin{array}{l}\text { Equipo élite }[F] \text { de la liga } \\
\text { profesional italiana } \\
\qquad(n=58)\end{array}$ & $\begin{array}{l}\text { Medición de } \mathrm{C}, \mathrm{T}, \mathrm{GH} \text { e } \\
\mathrm{IGF}-1 \text { séricos durante } \\
\text { la temporada de } \\
\text { entrenamiento } \\
\text { (septiembre - mayo) }\end{array}$ & $\begin{array}{l}\text { Frente a las concentraciones séricas } \\
\text { establecida en la práctica clínica, las } \\
\text { deportistas mostraron niveles elevados de: } \\
\mathrm{C}(p<0,01) \text {; IGF-1 }(p<0,001) \text {; } \mathrm{T}(p<0,05) \text {; } \\
\mathrm{GH}(p<0,001)\end{array}$ \\
\hline $\begin{array}{l}\text { Edwards \& Kurlander } \\
\qquad(2010)\end{array}$ & $\begin{array}{l}\text { Jugadoras }[F] \text { del equipo } \\
\text { de voleibol de la } \\
\text { Universidad de Emory, } \\
\text { EEUU }(n=15)\end{array}$ & \begin{tabular}{lrr} 
Medición de C y & \multicolumn{1}{c}{} \\
previo & al \\
calentamiento, & 30 \\
minutos después & de \\
iniciado & el \\
calentamiento, y & 80 \\
minutos posterior al \\
inicio del partido
\end{tabular} & $\begin{array}{l}\text { Los niveles de C y T aumentaron durante el } \\
\text { calentamiento. } \\
\text { Las jugadoras que participaron en el partido } \\
\text { mostraron un incremento significativo }(p< \\
0,01) \text { en los niveles C y T al terminar el } \\
\text { partido frente al calentamiento. Por el } \\
\text { contrario, quienes no jugaron, mostraron } \\
\text { una reducción en C y T frente al } \\
\text { calentamiento }\end{array}$ \\
\hline Eliakim et al. (2013) & $\begin{array}{l}\text { Jugadoras }[F] \text { élite del } \\
\text { equipo israelí de voleibol } \\
\text { categoría juvenil } \\
\qquad(n=13)\end{array}$ & $\begin{array}{l}\text { Medición de C, GH, } \\
\text { IGF-1, IGFBP3 e IL-6 } \\
\text { previo y posterior a una } \\
\text { práctica de } 60 \text { minutos } \\
\text { de voleibol. Toma de } \\
\text { muestras en dos } \\
\text { escenarios: antes y } \\
\text { después de } 7 \text { semanas } \\
\text { de entrenamiento }\end{array}$ & $\begin{array}{l}\text { Frente a la primera sesión de } 60 \text { minutos, } \\
\text { en la segunda (posterior a las } 7 \text { semanas de } \\
\text { entrenamiento), se observó: } \\
\text { aumento de } \mathrm{GH}(p<0,05) \text {, disminución de } \\
\mathrm{C}(p<0,05) \text {, y ninguna variación en los } \\
\text { niveles de IGF-1 }\end{array}$ \\
\hline Nemet et a & $\begin{array}{l}\text { Jugadores [M] élite del } \\
\text { equipo israelí de voleibol } \\
\text { categoría juvenil } \\
\qquad(n=14)\end{array}$ & $\begin{array}{l}\text { Medición de C, T, GH, } \\
\text { IGF-1, IGFBP3 e IL-6 } \\
\text { previo y posterior a una } \\
\text { práctica de } 60 \text { minutos } \\
\text { de voleibol. Toma de } \\
\text { muestras en dos } \\
\text { escenarios: antes y } \\
\text { después de } 7 \text { semanas } \\
\text { de entrenamiento }\end{array}$ & $\begin{array}{l}\text { En la primera sesión de } 60 \text { minutos, se } \\
\text { observó un aumento de } \mathrm{GH} \text { y } \mathrm{T} \text { luego del } \\
\text { ejercicio. } \\
\text { Posterior a las } 7 \text { semanas de } \\
\text { entrenamiento, se observó: } \\
\text { aumento de } \mathrm{GH}(p<0,05) \text { y ninguna } \\
\text { variación significativa en los niveles de IGF- } \\
1 \text { y C, luego de la sesión de ejercicio }\end{array}$ \\
\hline Antonelli et al. (2007) & $\begin{array}{l}\text { Jugadoras }[F] \text { de voleibol } \\
\text { categoría juvenil }(n=15) \text { y } \\
\text { mujeres sedentarias } \\
\qquad(n=14)\end{array}$ & $\begin{array}{l}\text { Medición de los niveles } \\
\text { basales de IGF-1 } \\
\text { (saliva y suero) y GH } \\
\text { (suero) posterior a una } \\
\text { sesión de juego }\end{array}$ & $\begin{array}{l}\text { Los niveles de IGF-1 en saliva fueron } \\
\text { menores en jugadoras de voleibol frente a } \\
\text { mujeres sedentarias }(p<0,001) \text {, mientras } \\
\text { que los niveles séricos de IGF-1 y GH no } \\
\text { mostraron una diferencia significativa }\end{array}$ \\
\hline Chaari et al. (2012) & $\begin{array}{l}130 \text { niños [M] en edad } \\
\text { prepuberal divididos en } \\
\text { tres grupos: } \\
\text { (1) Jugadores de voleibol } \\
\text { de alto rendimiento } \\
\text { ( } n=40) \\
\text { (2) Jugadores de voleibol } \\
\text { de bajo rendimiento } \\
(n=40) \\
\text { (3) Sujetos sedentarios } \\
(n=50)\end{array}$ & $\begin{array}{l}\text { Medición de los niveles } \\
\text { séricos basales de GH, } \\
\text { IGF-1, C, IGFBP3 y } \\
\text { marcadores de } \\
\text { recambio óseo }\end{array}$ & $\begin{array}{l}\text { Los niveles de IGF-1, C y GH mostraron } \\
\text { ser significativamente más elevados en } \\
\text { jugadores de alto rendimiento (1) frente a } \\
\text { los demás grupos }((2) \text { y }(3))\end{array}$ \\
\hline
\end{tabular}


Continuación tabla 1.

\begin{tabular}{|c|c|c|c|}
\hline Autor(es) (año) & Población de estudio & Diseño experimental & Resultados relevantes \\
\hline Horta et al. (2019): & $\begin{array}{l}\text { Jugadores }[\mathrm{M}] \text { de voleibol } \\
\text { de categoría mayores en } \\
\text { la Súper Liga brasilera de } \\
\text { voleibol } \\
(\mathrm{n}=12)\end{array}$ & $\begin{array}{l}\text { Medición de los niveles } \\
\text { séricos de T, C y } \\
\text { determinación de la } \\
\text { razón T/C durante la } \\
\text { 2nda, 4ta y 6ta semana } \\
\text { de entrenamiento de } \\
\text { alta carga }\end{array}$ & $\begin{array}{l}\text { Los niveles séricos de T aumentaron } \\
\text { posterior al periodo de entrenamiento (6ta } \\
\text { semana) }(p<0,05) \text {. } \\
\text { Los niveles de } C \text { sérico no mostraron una } \\
\text { variación significativa }\end{array}$ \\
\hline Mazon et al. (2013): & $\begin{array}{l}\text { Jugadores [M] élite de } \\
\text { voleibol categoría } \\
\text { mayores en la ciudad de } \\
\text { Sao Paolo, Brasil } \\
(n=32)\end{array}$ &  & $\begin{array}{l}\text { Los niveles de } C \text { séricos disminuyeron al } \\
\text { final de la temporada de competencia }(p< \\
0,05) \text { frente al inicio. La concentración } \\
\text { plasmática de T y la razón T/C aumentaron } \\
\text { significativamente }(p<0,05)\end{array}$ \\
\hline $\begin{array}{l}\text { Peñailillo et al. } \\
\text { (2018): }\end{array}$ & $\begin{array}{c}\text { Jugadores del equipo } \\
\text { masculino }(n=12) \text { y } \\
\text { femenino }(n=12) \text { nacional } \\
\text { de Chile, categorias } \\
\text { mayores }\end{array}$ & $\begin{array}{l}\text { Medición de C y T en } \\
\text { saliva antes y después } \\
\text { de cada partido. } \\
\text { Se evaluaron r } \quad 3 \\
\text { partidos, para un total } \\
\text { de } 6 \text { muestras }\end{array}$ & $\begin{array}{l}\text { Los niveles de } C \text { basales, previo al partido, } \\
\text { fueron mayores en las mujeres }(p<0,001) \text {. } \\
\text { La T basal fue mayor en hombres }(p< \\
0,001) \text {. } \\
\text { La T disminuyó en el tercer partido para los } \\
\text { hombres, pero aumentó significativamente } \\
\text { en las mujeres }(p<0,01)\end{array}$ \\
\hline Turgut et al. (2003) & $\begin{array}{c}\text { Niños }(n=14) \text { y niñas } \\
(n=13) \text { en edad } \\
\text { prepuberal }\end{array}$ & $\begin{array}{l}\text { Medición de los niveles } \\
\text { urinarios de IGF-1, } \\
\text { IGFBP3, creatinina, } \\
\text { posterior a una sesión } \\
\text { de práctica de voleibol } \\
\text { de } 2 \text { horas }\end{array}$ & $\begin{array}{l}\text { Los niveles de IGF-1 aumentaron } \\
\text { significativamente posterior a la sesión de } \\
\text { práctica en los } 27 \text { sujetos }(p<0,01) \text { y en la } \\
\text { misma proporción, tanto en hombres }(p< \\
0,01) \text { como en mujeres }(p<0,01)\end{array}$ \\
\hline
\end{tabular}

Abreviaciones: C: Cortisol; T: Testosterona; GH: hormona de crecimiento; IGF-1: Factor de crecimiento similar a la insulina tipo 1; IGFBP3: Proteína 3 enlazante al factor de crecimiento similar a la insulina; IL-1: Interleucina-1; IL-6: Interleucina-6. [M]: Sexo masculino y [F]: Sexo femenino.

El eje hipotálamo-hipófisis-adrenal (HHA) integra los mecanismos reguladores de la producción de cortisol y, por tanto, constituye un importante blanco de estudio para la endocrinología aplicada al deporte. El cortisol es una hormona hiperglicemiante, lipolítica y estimulante de la proteólisis muscular (Christiansen et al. 2007). Así, este glucocorticoide cumple una función catabólica, que favorece el aumento de la biodisponibilidad de sustratos para suplir la demanda energética, generada en condiciones de estrés (Pulopulos et al. 2020).

Dos factores principales modulan la secreción de cortisol durante la actividad física: la intensidad (VOmax) y la duración del ejercicio. En condiciones de estrés físico, la activación del eje HHA en atletas difiere de aquella observada en sujetos sedentarios (Constantini \& Hackney, 2013). Se han propuesto distintos mecanismos subyacentes a este fenómeno, entre estos, cambios en los patrones de actividad neuroendocrina, mediados por neuropéptidos como las endorfinas (Schwarz \& Kindermann, 1990), y variaciones en la sensibilidad a los glucocorticoides, tanto a nivel central como periférico (Duclos et al. 2003).

Los estudios recolectados en la presente revisión muestran que los voleibolistas exhiben niveles basales de cortisol elevados frente a la población sedentaria sana (Roli et al. 2018) y, a su vez, parecen variar de acuerdo con el tiempo de juego en partidos y la categoría de los mismos (Edwards \& Kurlander, 2010; Chaari et al. 2012). Por otra parte, al finalizar las temporadas de competencia deportiva, el cortisol parece disminuir significativamente frente a los elevados niveles que se observan al inicio de esta (Mazon et al. 2013).

Diversas investigaciones sugieren que el incremento en la concentración de cortisol, previo a competencias deportivas, se asocia con ansiedad y bajo desempeño (Papacosta et al. 2016; Cintineo \& Arent, 2019). A la fecha, no se ha reportado el primer estudio de este tipo en jugadores de voleibol, y aunque existe evidencia 
que el entrenamiento puede reducir el incremento de cortisol previo a sesiones de juego (Eliakim et al. 2013), otros estudios afirman que la respuesta del cortisol a las competencias deportivas no difiere antes y después de la temporada de entrenamiento (Nemet et al. 2012; Horta et al. 2019).

Contrario a la acción catabólica del cortisol, la respuesta hormonal, mediada por la testosterona, establece una respuesta anabólica a la actividad física. La testosterona favorece el crecimiento de la masa muscular (Kelly \& Jones, 2013), la eritropoyesis (Shahani et al. 2009) y parece aumentar la sensibilidad a la insulina (Simoni \& Huhtaniemi, 2017). Por otro lado, la testosterona se ha asociado con patrones de comportamiento como la agresividad (Delgado et al. 2020) y la competitividad (Casto \& Edwards, 2016). En la práctica deportiva, la testosterona potencia el rendimiento físico, a tal punto, que el uso de esteroides anabólicos sintéticos se ha prohibido en las competencias deportivas (Wood et al. 2012).

La testosterona hace parte de un grupo de esteroides, denominados andrógenos. Estas hormonas se encuentran en concentraciones significativamente mayores en hombres, y los estudios han mostrado que elevados niveles circulantes de andrógenos suponen una ventaja ergogénica del 8-12 \%, en individuos de sexo masculino (Handelsman et al. 2018). A raíz de este dimorfismo sexual, se han propuesto estrictos rangos de testosterona sérica en atletas femeninas, a tal punto, que se plantea que las mujeres con hiperandrogenismo no controlado deben presentar niveles circulantes de andrógenos menores a $5 \mathrm{nmol} / \mathrm{L}$ para permitir su participación en competencias deportivas (Handelsman et al. 2018).

Los estudios recopilados en esta investigación muestran que existe una variación en los niveles de testosterona, tanto en atletas masculinos como femeninos, durante sesiones de práctica deportiva. Similaral cortisol, la testosterona es alta en voleibolistas de sexo femenino frente a los valores de referencia de sujetos sedentarios (Roli et al. 2018) y el aumento de esta hormona, depende del tiempo de juego (aunque el incremento es mayor en hombres). Adicionalmente, la concentración de testosterona parece ser mayor al finalizar la temporada de competencia (Mazon et al. 2013), pero la evidencia es inconclusa frente al efecto de la temporada de entrenamiento en los cambios en los niveles séricos de testosterona. Horta et al. (2019) evidenciaron que el aumento de testosterona durante una sesión de práctica es mayor luego de 6 semanas de entrenamiento; por el contrario, Nemet et al. (2012) encontraron un mayor incremento previo a la temporada de entrenamiento. Así, evaluar el efecto del entrenamiento de voleibol sobre el incremento de la testosterona durante una sesión de juego, es un aspecto que permanece aún sujeto a futuros estudios.

La hormona de crecimiento $(\mathrm{GH})$ es un polipéptido hipofisiario con efecto hiperglicemiante, lipolítico y estimulante de la síntesis de proteínas (Bergan-Roller et al. 2018). En el hígado, la GH estimula la producción del factor de crecimiento similar a la insulina tipo 1 (IGF-1). A pesar de su acción hipoglicemiante (opuesto a la GH), el IGF-1 actúa de forma complementaria a la $\mathrm{GH}$, favoreciendo el crecimiento lineal en etapas de desarrollo, y en edades pospuberales generando un aumento de la masa muscular y una redistribución de la grasa visceral hacia la periferia (Nicholls \& Holt, 2016). Este efecto anabólico ha llevado a la administración excesiva de $\mathrm{GH}$ recombinante por parte de atletas y, en consecuencia, a su clasificación como droga de abuso en el deporte (Baumann, 2012). Independientemente a la administración exógena, la secreción de GH aumenta durante la actividad física (Kanaley, 2008); es por esto, que fomentar la práctica deportiva en niños en etapa de desarrollo es una estrategia adecuada y pertinente para potenciar el crecimiento somático. En concordancia, gran parte de los estudios incluidos en esta revisión, indagan en los niveles circulantes de GH e IGF-1, en sujetos adolescentes o en edades prepuberales.

La evidencia recolectada muestra que el aumento de GH es más significativo posterior a la temporada de entrenamiento; sin embargo, la magnitud del incremento de IGF-1 parece no variar luego de periodos de entrenamiento (Eliakim et al. 2013; Nemet et al. 2012). Adicionalmente, la elevación de los niveles de GH e IGF-1 parece ocurrir de forma similar en sujetos en edad prepuberal, ya sean de sexo masculino o femenino (Turgut et al. 2003). En adultos, por otra parte, los niveles de GH e IGF-1 son significativamente mayores frente a los valores establecidos para la edad correspondiente. Finalmente, Chaari et al. (2012) reportaron que el aumento de GH e IGF-1 es mayor en jugadores de voleibol de alto rendimiento en edad prepuberal y, por el contrario, Antonelli et al. (2007) determinaron que los niveles salivales de IGF-1 son menores en jugadoras de voleibol frente a 
sujetos sedentarios. Por lo tanto, evaluar la variación de la concentración de IGF-1 en voleibolistas en edad prepuberal en relación con su categoría de juego y en comparación con sujetos sedentarios, está sujeto a futuras investigaciones.

Adicional a las hormonas anteriormente discutidas, se evidenció la evaluación de otros marcadores séricos. En primer lugar, la interleucina 6 (IL-6) fue estudiada en voleibolistas por Nemet et al. (2012) y Eliakim et al. (2013), quienes hallaron que la IL-6 aumentaba significativamente posterior a una sesión de práctica de voleibol, y que este incremento era mayor, al finalizar la temporada de entrenamiento. El aumento de las citoquinas pro-inflamatorias, como la IL-6, se ha asociado al estrés del ejercicio físico (Paolucci et al. 2018); sin embargo, la evidencia también apunta a que existe un aumento considerable de citoquinas antiinflamatorias durante el ejercicio moderado (GómezBanoy et al. 2016). La evaluación de los niveles de catecolaminas (Mazon et al. 2013) y de creatinina (Turgut et al. 2003) mostró un aumento después de las sesiones de práctica de voleibol. Por otro lado, los marcadores de recambio óseo no mostraron variación alguna (Chaari et al. 2012). Por último, los niveles circulantes de IGFBP3 no aumentaron posterior a una sola sesión de voleibol (Turgut et al. 2003; Eliakim et al. 2013), sin embargo, parecen incrementar después de la temporada de entrenamiento en jugadores de alto rendimiento (Chaari et al. 2012; Nemet et al. 2012).

\section{CONCLUSIONES}

La presente investigación desarrolló una revisión de los estudios referentes a la respuesta hormonal adaptativa durante la práctica del voleibol, en jugadores de sexo masculino y femenino, en diferentes categorías de juego. A partir de los resultados se evidencia que, en respuesta a las sesiones de práctica de voleibol de piso, los deportistas exhiben un incremento en los niveles de cortisol, testosterona y GH. Este aumento está sujeto al tiempo de juego posterior al calentamiento y a la categoría de los voleibolistas. Aunque existe evidencia contradictoria de los cambios en los niveles de IGF-1 e IGFBP3, en respuesta a la práctica del voleibol, también se reportó aumento de catecolaminas, IL-6 y creatinina, pero ningún cambio en marcadores de recambio óseo.

Finalmente, los estudios sugieren que el entrenamiento físico podría ayudar a adaptar al organismo a regular la respuesta endocrina previo a una competencia deportiva. Los resultados apuntan a que, posterior al periodo de entrenamiento $y$ acondicionamiento físico, el cambio neto de cortisol es menor y el de testosterona es mayor, durante los periodos de campeonato. Dado que la evidencia es poco conclusiva, dilucidar la modulación de la respuesta endocrina durante una competencia de voleibol de piso, en respuesta a temporadas de entrenamiento y acondicionamiento físico, está sujeto a futuras investigaciones.

Conflicto de interés: Los autores reportan no tener ningún conflicto de interés.

\section{REFERENCIAS}

1. ANTONELLI, G.; CAPPELLIN, E.; GATTI, R.; CHIAPPIN, S.; SPINELLA, P.; DE PALO, E. 2007. Measurement of free IGF-I saliva levels: Perspectives in the detection of GH/IGF axis in athletes. Clin. Biochem. 40(8):545-550. https:// doi.org/10.1016/j.clinbiochem.2007.01.014

2. BAUMANN, G. 2012. Growth hormone doping in sports: a critical review of use and detection strategies. Endocr. Rev. 33(2):155-186. https://doi.org/10.1210/er.2011-1035

3. BERGAN-ROLLER, H.; SHERIDAN, M. 2018. The growth hormone signaling system: insights into coordinating the anabolic and catabolic actions of growth hormone. Gen. Comp. Endocrinol. 258:119-133. https:// doi.org/10.1016/j.ygcen.2017.07.028

4. BUDDE, H.; WEGNER, M.; SOYA, H.; VOELCKERREHAGE, C.; MCMORRIS, T. 2016. Neuroscience of exercise: neuroplasticity and its behavioral consequences. Neural Plast. 2016:1-3. https://doi.org/10.1155/2016/3643879

5. CACCESE, C.; JONES, S.; RAMESH, M.; YU, A.; BROSSARD-RACINE, M.; NGUYEN, T. 2021. Role of testosterone: cortisol ratio in ageand sex-specific cortico-hippocampal development and cognitive performance. J. Dev.Orig. Health Dis. 1-11. https:// doi.org/10.1017/S204017442100012X 
6. CASTO, K.; EDWARDS, D. 2016. Testosterone, cortisol, andhumancompetition. Horm. Behav.82:21-37. https://doi.org/10.1016/j.yhbeh.2016.04.004

\section{CASTRO-SEPÚLVEDA, M.; CANCINO, J.; FERNÁNDEZ-} VERDEJO, R.; PÉREZ-LUCO, C.; JANNAS-VELA, S.; RAMÍREZ-CAMPILLO, R.; DEL COSO, J.; ZBINDEN-FONCEA, H.2019. Basalserumcortisol and testosterone/cortisol ratio are related to rate of $\mathrm{Na}+$ lost during exercise in elite soccer players. Int J Sport Nutr Exe. 29(6):658-663. https://doi.org/10.1123/ijsnem.2019-0129

8. CHAARI, H.; ZOUCH, M.; DENGUEZLI, M.; BOUAJINA, E.; ZAOUALLI, M.; TABKA, Z. 2012. A high level of volleyball practice enhances bone formation markers and hormones in prepubescent boys. Biol. Sport. 29(4):303-309.

9. CHRISTIANSEN, J.; DJURHUUS, C.; GRAVHOLT, C.; IVERSEN, P.; CHRISTIANSEN, J.; SCHMITZ, O.; WEEKE, J.; JORGENSEN, J.; MOLLER, N. 2007. Effects of cortisol on carbohydrate, lipid, and protein metabolism: studies of acute cortisol withdrawal in adrenocortical failure. J. Clin. Endocrinol. Metab. 92(9):3553-3559. https://doi.org/10.1210/jc.2007-0445

10. CINTINEO, H.; ARENT, S. 2019. Anticipatory salivary cortisol and state anxiety before competition predict match outcome in division I collegiate wrestlers. J. Strength Cond. Res. 33(11):2905-2908. https://doi.org/10.1519/JSC.0000000000003376

11. CLOSS, B.; BURKETT, C.; TROJAN, A.; BROWN, S.; MULCAHEY, M. 2020. Recovery after volleyball: a narrative review. Phys Sportsmed. 48(1):8-16. https://doi.or g/10.1080/00913847.2019.1632156

12. CONSTANTINI, N.; HACKNEY, A. 2013. Endocrinology of physical activity and sport. Humana Press, New York. 558p.

13. DELGADO, P.; MAYA-ROSERO, E.; FRANCO, M.; MONTOYA-OVIEDO, N.; GUATIBONZA, R.; MOCKUS, I. 2020. Testosterona y homicidio: aspectos neuroendocrinos de la agresión. Rev. Fac. Med. 68(2). https://doi.org/10.15446/revfacmed.v68n2.73237
14. DUCLOS, M.; GOUARNE, C.; BONNEMAISON, D. 2003. Acute and chronic effects of exercise on tissue sensitivity to glucocorticoids. J. Appl. Physiol. 94(3):869-875. https://doi.org/10.1152/japplphysiol.00108.2002

15. EDWARDS, D.; KURLANDER, L. 2010. Women's intercollegiate volleyball and tennis: effects of warm-up, competition, and practice on saliva levels of cortisol and testosterone. Horm. Behavior. 58(4):606-613. https://doi.org/10.1016/j.yhbeh.2010.06.015

16. ELIAKIM, A.; PORTAL, S.; ZADIK, Z.; MECKEL, Y.; NEMET, D. 2013. Training reduces catabolic and inflammatory response to a single practice in female volleyball players. J. Strength Cond. Res. 27(11):3110-3115. https:// doi.org/10.1519/JSC.0b013e31828d61d1

17. GÓMEZ-BANOY, N.; MOCKUS, I.; ARANZÁLEZ, L.; ZAMBRANO, J. 2016. Changes to circulating inflammatory cytokines in response to moderate exercise. J. Sport Med. Phys. Fit. 56(1-2):100-104.

18. HACKNEY, A.; LANE, A. 2015. Exercise and the regulation of endocrine hormones. Prog. Mol. Biol. Transl. Sci. 135:293-311. https://doi.org/10.1016/bs.pmbts.2015.07.001

19. HANDELSMAN, D.; HIRSCHBERG, A.; BERMON, S. 2018. Circulating testosterone as the hormonal basis of sex differences in athletic performance. Endocr. Rev. 39(5):803-829. https://doi.org/10.1210/er.2018-00020

20. HILL, E.; ZACK, E.; BATTAGLINI, C.; VIRU, M.; VIRU, A.; HACKNEY, A. 2008. Exercise and circulating cortisol levels: the intensity threshold effect. J. Endocrinol. Investig. 31(7):587-591. https://doi.org/10.1007/BF03345606

21.HÓMEZ, J.;PÉREZ, O.; VERA, D. 2017. Caracterización de la jugadora de voleibol de Bogotá en talla, peso, alcance en remate y alcance en bloqueo en las categorías infantil, menores y juvenil. R. Actividad Fis. y Deporte. 3(1):71-79. 
22. HORTA, T.; MAURÍCIO, G.; BARA, B.; FILHO, D.; COIMBRA, R.; MIRANDA, R.; WERNECK, Z. 2019. Training load, physical performance, biochemical markers, and psychological stress during a short preparatory period in Brazilian elite male volleyball players. J. Strength Cond. Res. 33(12):3392-3399. https://doi.org/10.1519/JSC.0000000000002404

23. KANALEY, J. 2008. Growth hormone, arginine and exercise. Curr. Opin. Clin. Nutr. Metab. Care. 11(1):50-54. https://doi.org/10.1097/MCO.0b013e3282f2b0ad

24. KELLY, D.; JONES, T. 2013. Testosterone: a metabolic hormone in health and disease. J. Endocrinol. 217(3):R25-45. https://doi.org/10.1530/JOE-12-0455

25. KRAEMER, W.; RATAMESS, N.; HYMER, W.; NINDL, B.; FRAGALA, M. 2020. Growth hormone(s), testosterone, insulin-like growth factors, and cortisol: roles and integration for cellular development and growth with exercise. Front. Endocrinol. 11:33. https://doi.org/10.3389/fendo.2020.00033

26. MAZON, J.; GASTALDI, A.; DI SACCO, T.; COZZA, I.; DUTRA, S.; SOUZA, H. 2013. Effects of training periodization on cardiac autonomic modulation and endogenous stress markers in volleyball players: models of periodization and stress markers. Scand. J. Med. Sci. Spor. 23(1):114-120. https://doi. org/10.1111/j.1600-0838.2011.01357.x

27. MCGEE, S.; HARGREAVES, M. 2020. Exercise adaptations: molecular mechanisms and potential targets for therapeutic benefit. Nat. Rev. Endocrinol. 16(9):495-505. https:// doi.org/10.1038/s41574-020-0377-1

28. MEEUSEN, R.; DUCLOS, M.; FOSTER, C.; FRY, A.; GLEESON, M.; NIEMAN, D.; RAGLIN, J.; RIETJENS, G.; STEINACKER, J.; URHAUSEN, A. 2013. Prevention, diagnosis, and treatment of the overtraining syndrome: joint consensus statement of the European College of Sport Science and the American College of Sports Medicine.Med.Sci.SportsExerc.45(1):186-205. https://doi.org/10.1249/MSS.0b013e318279a10a
29. MOHER, D.; LIBERATI, A.; TETZLAFF, J.; ALTMAN, D. 2009. Reprint-Preferred reporting items for systematic reviews and meta-analyses: the prisma statement. Phys. Ther. 89(9):873-880. https://doi.org/10.1093/ptj/89.9.873

30. NEMET, D.; PORTAL, S.; ZADIK, Z.; PILTZ-BURTEIN, R.; ADLER-PORTAL, D.; MECKEL, Y.; ELIAKIM, A. 2012. Training increases anabolic response and reduces inflammatory response to a single practice in elite male adolescent volleyball players. J. Pediatr. Endocrinol. Metab. 25:(910). https://doi.org/10.1515/jpem-2012-0094

31. NICHOLLS, A.; HOLT, R. 2016. Growth hormone and insulin-like growth factor-1. Front. Horm. Res. In: Lanfranco, F.; Strasburger, C.J. (eds.). Sports Endocrinology. 47:101114. https://doi.org/10.1159/000445173

32. NUNES-SILVA, A.; CARVALHO, G.; MASSOTE, D.; NEVES, L.; SALVIANO DE FARÍA, M.; SIMOES, E.; SILVA, A. 2017. Physical exercise and ace2angiotensin-(1-7)-mas receptor axis of the renin angiotensin system. Protein Pept. Lett. 24(9). https://doi.org/10.2174/0929866524666170728151401

33. PAOLUCCI, E.; LOUKOV, D.; BOWDISH, D.; HEISZ, J. 2018. Exercise reduces depression and inflammation but intensity matters. Biol. Psychol. 133:79-84. https://doi. org/10.1016/j.biopsycho.2018.01.015

34. PAPACOSTA, E.; NASSIS, G.; GLEESON, M. 2016. Salivary hormones and anxiety in winners and losers of an international judo competition. J. Sports. Sci. 34(13):1281-1287. https://doi.org/10.1080/02640414.2015.1111521

35. PEÑAILILLO, L.; ESCANILLA, F.; JURY, E.; CASTRO-SEPÚLVEDA, M.; DELDICQUE, L.; ZBINDEN-FONCEA, H. 2018. Differences in salivary hormones and perception of exertion in elite women and men volleyball players during tournament. J. Sport Med. Phys. Fit. 58(11):1688-1694. https://doi. org/10.23736/S0022-4707.17.07681-2

36. PULOPULOS, M.; BAEKEN, C.; DE RAEDT, R. 2020. Cortisol response to stress: the role of expectancy and anticipatory stress 
regulation. Horm. Behav. 117:104587. https://doi.org/10.1016/j.yhbeh.2019.104587

37. ROLI, L.; DE VINCENTIS, S.; ROCCHI, M.; TRENTI, T.; DE SANTIS, M.; SAVINO, G. 2018. Testosterone, cortisol, HGH, and IGF-1 levelsinan Italianfemale elite volleyball team. Health Sci. Rep. 1(4):e32. https://doi.org/10.1002/hsr2.32

38.SCHWARZ, L.; KINDERMANN, W.1990. $\beta$-Endorphin, adrenocorticotropic hormone, cortisol and catecholamines during aerobic and anaerobic exercise. Eur. J. Appl. Physiol. 61(3-4):165-171. https://doi.org/10.1007/BF00357593

39. SHAHANI, S.; BRAGA-BASARIA, M.; MAGGIO, M.; BASARIA, S. 2009. Androgens and erythropoiesis: past and present. J. Endocrinol. Investig. 32(8):704-716. https://doi.org/10.1007/BF03345745

40. SIMONI, M.; HUHTANIEMI, I. 2017. Endocrinology of the testis and male reproduction. Springer International Publishing (Italy). 1364p. https://doi.org/10.1007/978-3-319-44441-3

41. SOUGLIS, A.; BOGDANIS, G.; GIANNOPOULOU, I.; PAPADOPOULOS, CH.; APOSTOLIDIS, N. 2015. Comparison of inflammatory responses and muscle damage indices following a soccer, basketball, volleyball and handball game at an elite competitive level. Res. Sports Med. 23(1):59-72. https:// doi.org/10.1080/15438627.2014.975814

42. TURGUT, G.; KAPTANOGLU, B.; TURGUT, S.; GENÇ, O.; TEKINTÜRK, S. 2003. Influence of acute exercise on urinary protein, creatinine, insulin-like growth factor-I (IGF-I) and IGF binding protein-3 concentrations in children. Tohoku J. Exp. Med. 201(3):165-170. https://doi.org/10.1620/tjem.201.165

43. URHAUSEN, A.; HOLGER, G.; KINDERMANN, W. 1995. Blood hormones as markers of training stress and overtraining. Sports Med. 20(4):251-276. https://doi. org/10.2165/00007256-199520040-00004

44. WOOD, R.; STANTON, S. 2012. Testosterone and sport: current perspectives. Horm. Behav. 61(1):147-155. https://doi.org/10.1016/j.yhbeh.2011.09.010

45. ZOUHAL, H.; JACOB, C.; DELAMARCHE, P.; GRATASDELAMARCHE, A. 2008. Catecholamines and the effects of exercise, training and gender. Sports Med. 38(5):401-423. https://doi. org/10.2165/00007256-200838050-00004 